\title{
A complete framework for the synthesis of powered floor systems
}

\author{
Stefano Seriani, Eric Medvet, Sergio Carrato, and Paolo Gallina
}

\begin{abstract}
One of the most pressing issues in the field of mobile robotics is that of power delivery. By being fundamentally unattached to the environment, these systems often employ batteries. However, this can have some limitations related to cost, battery life etc.; a promising approach is that of powered floors, where a specially designed contact array is connected to the robot, and slides on a surface in which conducting bands are laid out, providing a continuous and uninterrupted electrical connection. In this paper, we provide a complete framework for the analysis and synthesis of these systems, to be used in the field of mobile robotics. We study the problem both in terms of feasibility and in quantitative terms; in particular, we illustrate a methodology related to n-sided polygons which takes into account a fuzzy representation of the bands, useful to analyze tolerances in the bands boundaries or in the position of the individual brushes. We support the theoretical framework with both numerical and experimental campaigns, and compare the results with existing state-of-the art solutions. Finally, we discuss possible further developments.
\end{abstract}

Index Terms-Powered floor, wheeled robots, industrial robots, mechanism design.

\section{INTRODUCTION}

The task of delivering electrical power to mobile robots or, more generally, to mobile systems, continues to challenge many aspects of the world of transportation, automation and logistics. The vast majority of electrical mobility traditionally relies on batteries, including road transportation, industrial logistics, and mobile robots [1]. Perhaps the most endemic solution that does not involve the use of energy storage is that of the electrified railroad system, which employs sliding contacts mounted on pantographs, closing the circuit with aerial cable conductors [2], [3]. Alternative, less common solutions are conductors embedded in the road, e.g., the Ansaldo-Breda project TramWave [4].

In the field of robotics, in recent years, the industry has seen incremental adoption of Autonomous Ground Vehicles (AGVs) mainly as a means of internal logistics. However, these systems rely almost completely on batteries for their energetic requirements, causing severe downtime due to charge time [5], [6]. For this reason, continuous charging methodologies and direct power delivery have been proposed [7]. The most common approach found in mobile robots literature is that of wireless energy delivery through the use of resonating

The authors are with the Department of Engineering and Architecture (DIA), University of Trieste, 34127 Trieste, Italy. (email: sseriani@units.it, emedvet@units.it, carrato@units.it,pgallina@units.it)

Corresponding author: Stefano Seriani, sseriani@units.it, Department of Engineering and Architecture, University of Trieste, via A. Valerio 6/1, 34127 Trieste, Italy.

Manuscript received ... coils [8], [9]. The same concept is found in the field of electrical road transportation [10], [11], [12], [13]. Several surveys are available on the recent advancements in the field of wireless power transfer [14], [15], [16]. In particular, Jang et al. discuss on the current state-of-the-art of these systems, highlighting the efficiency as an advantage, while costs of the infrastructure can prove high.

Other approaches rely on the logistics of power delivery, rather than the technology itself; this is the case of coordinated charging [17] and of energy logistics models [18]. Yet another mean to transfer energy to mobile robots is that of tethers [19], [20].

A common way of delivering energy to mobile robots is by means of sliding-contacts [21], [22]. This is the focus of the present paper. In general, this type of power delivery system allows for relative motion in a single direction, as is the case with slip-rings. However, some examples have been proposed that allow for bi-dimensional relative motion; in 2002, Watson et al. [23] apply a methodology-originally invented by C. Shannon at the AT\&T Bell Laboratories in 1950 - for continuous power delivery to small mobile swarm robots: this was based on sliding contacts. A more recent example is the Droplets platform [24]. That of swarm robotics is a field which shows remarkable affinity with the concept of powered floor [25], [26], [27]. Similarly, powered floors may be beneficial to evolutionary robotics, since they allow researchers and practitioners to run experiments for long times involving real robots, without interruptions for charging and physical obstacles impeding access to the robots [28], [29].

Powered floors based on sliding contacts are a flexible and inexpensive solution to provide continuous power to mobile robots; wireless power transfer systems, on the other hand, generally require costly infrastructure (i.e., the array of coils for the transmitter needs to be placed to cover the entire floor) and produce very high values of stray magnetic fields [14]. Ultimately, the application is key in the correct determination of the more suitable type of powered floor.

All other solutions (e.g., batteries, tethers, etc.) mentioned up to this point tend to severely hinder the freedom of movement of the vehicles, and are thus ill-suited either for continuous operation or in cluttered environments.

In this paper, we focus on a systematic study of the alternating bands sliding-contact based powered floor introduced by C. Shannon in the 1950s [23]; to the best of our knowledge, this is not available in current state-of-the-art. We provide a comprehensive framework for contacts patterns based on regular polygons of $n$ sides. We demonstrate that triangle- and rectangle-shaped patterns cannot always guarantee contact, 
and that Shannon's pattern itself has several limitations.

In Section II a comprehensive analysis of the concept of powered floor or powered surface is presented, along with the main factors that will be taken in considerations in the rest of the article; in Section III we present the mathematical framework which constitutes the backbone of the methodology; in Section IV we outline the methodology that can be used to design and analyze $n$-gon based powered floors; in Section V we present a full numerical and experimental campaign to both validate the methodology itself and to validate an octagon-based prototype; finally, in Section VI we present our concluding remarks.

\section{THE POWERED FLOOR}

Starting from the work of Shannon in the 1950s [23], the concept of powered floor has had limited development through the years. The idea revolves around using supernumerary, i.e., more than 2, sliding contacts, organized in a rigid contact array, that interacts with a floor made of alternating polarity bands, along with the electronics to switch between the bands in order to provide a constant source of power. We can define the contact array geometry as the spatial arrangement of the brushes with respect to a common frame of reference. The most common geometries for these constructs are the 3-contact equilateral triangle [24] and Shannon's 4-contact array. These can be seen in Fig. 1a, b and Fig. 1e, f respectively. The same figure shows a square configuration as well, for reference (Fig. 1c, d). The band geometry can be defined as the ratio between the width of the conducting bands and that of the insulator bands between them. In this respect, to achieve functionality, the geometry of the contact array and that of the bands need to be compatible.

It should be noted at this point that, in this work, we focus on patterns of arrays based on regular polygons; however, the mathematical framework which is highlighted in III and the related methodology are general and can be applied with minimal modifications to arrays of arbitrary geometry. For example, [30] describes a method for the automatic design of arrays of contacts based on evolutionary computation and built on a simplified version of the framework presented in this paper.

The most important aspect of the powered floor is consistent power delivery, i.e., power should be delivered with no interruptions. This problem is dependent on the geometry of the contact array and of the polarity bands. If we define a metric $\mu$ for the size of the components, then $\mu_{B}$ and $\mu_{C}$ are the sizes of the bands and contact-array respectively (e.g., the width of the bands, and the radius of the circular hull of all contact points). Note that the width of the insulator band is assumed constant. We can then state the problem as follows: "The powered floor-contact array pair is functional if there is at least one combination of $\mu_{B}$ and $\mu_{C}$ that guarantees a closed circuit, whatever the orientation and position of the contactarray with respect to the bands". In the following sections, these two quantities will be defined in terms of a mutual ratio, in order to keep a dimensionless approach.

In Fig. $1 \mathrm{~b}$ and $\mathrm{d}$ we show that some configurations (i.e., orientation and position relative to the floor) of the equilateral triangle and square produce non-closed circuits. This is true for whatever scale ratio $\mu_{C} / \mu_{B}$; in the case of the triangle, the reason being that if two points are on the insulation band, the only other point is not sufficient to close any circuit; in the case of the square, because of the inherent parallelism of its sides, if two points are on the insulation band the other two points will always be on the same polarity band, thus unable to provide a closed circuit. Note that this reasoning extends to parallelograms in general. In this case the conditions laid
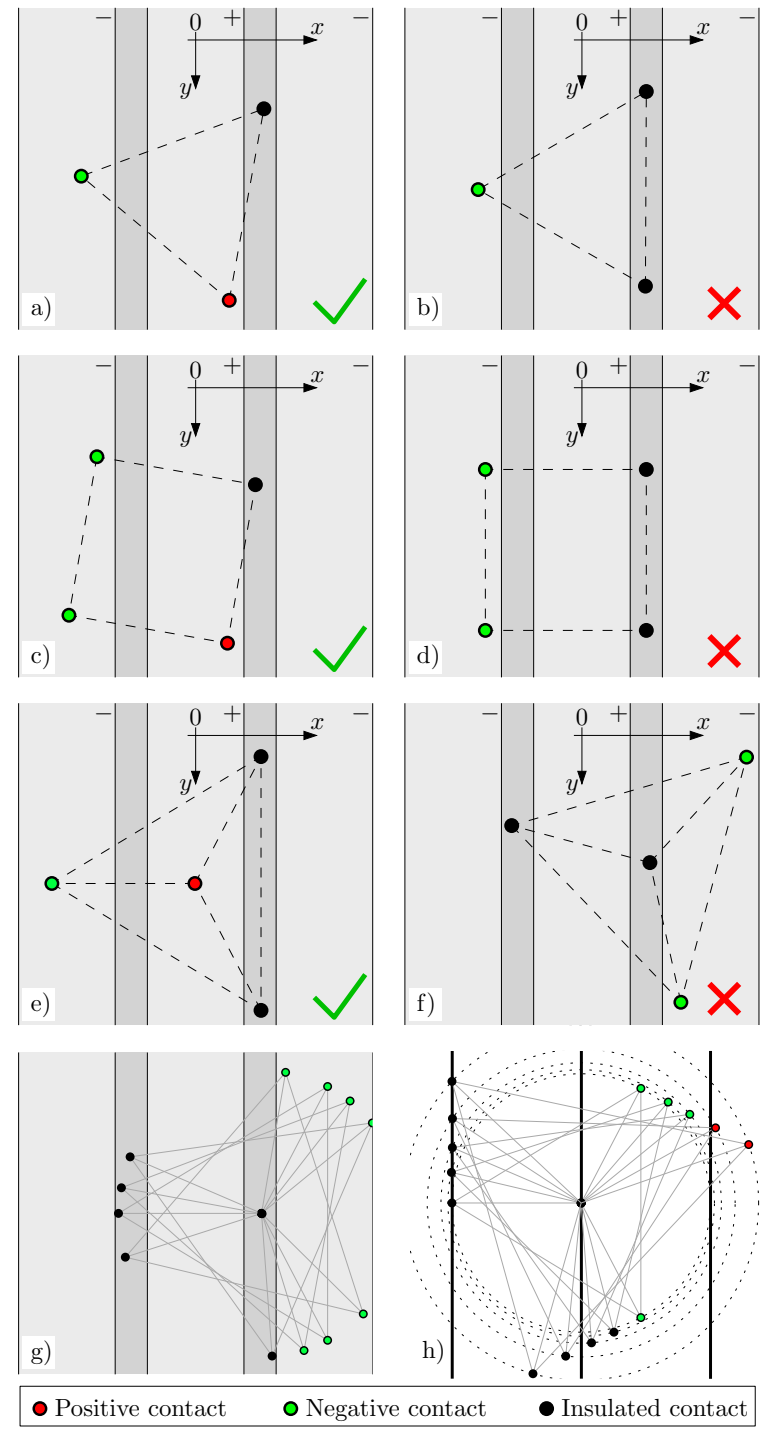

Fig. 1: Contact geometries. In a) a 3-brush geometry arranged as a regular triangle is shown to be connected both to the negative and positive poles. In b) the same geometry is shown but at a slightly different angle, showing only contact with the negative band. In c) a square brush geometry is shown that closes the circuit, whereas in d) the same is shifted by a slight amount, showing incomplete contact. In e) and in f) an embodiment of Shannon's design is illustrated, respectively in a complete contact configuration and one that is incomplete. In g) a set of constant size inadequate configurations are shown of Shannon's geometry, while in $f$ ) the same is done with thin insulation bands and varying $\mu_{C}$ dimension. 
down in the problem statement are not met, hence triangle and parallelogram geometries do not provide consistent electrical contact.

In Fig. If we show a special case of Shannon's geometry that does not provide a closed circuit in at least one configuration. Similarly, in $1 \mathrm{~g}$ a collection of orientations is shown to provide non adequate connection; finally, in $\mathrm{h}$ ) an illustration is shown of several configurations of $\mu_{B}$ and $\mu_{C}$ that produce non-adequate electrical contact. To best describe this, zerowidth insulation bands are used. Consequently, non-functional configurations such as these can be avoided by selecting a proper $\mu_{C} / \mu_{B}$ scale ratio.

While it can be shown that Shannon's geometry behaves adequately when the insulator bands are thin, we note that it falls short where these are wider. In fact, in this paper we illustrate how pentagons and higher polygons provide best results when insulator bands are very wide. A hint of this lies in the reasoning that a higher number of contact points has a higher probability of being in contact with both polarity bands. An example is shown in Fig. 2, where a pentagon is shown in a functional (a) and non-functional (b) configurations, which differ by their $\mu_{C} / \mu_{B}$ ratio; finally, a prototype is illustrated (c) of an 8-contact point system.

\section{MATHEMATICAL FRAMEWORK}

The geometry of the contact points and of the polarity bands deeply influences the performance of the powered floor and of the delivery system in general, i.e., the functionality and robustness of the system. Hence, in order to provide

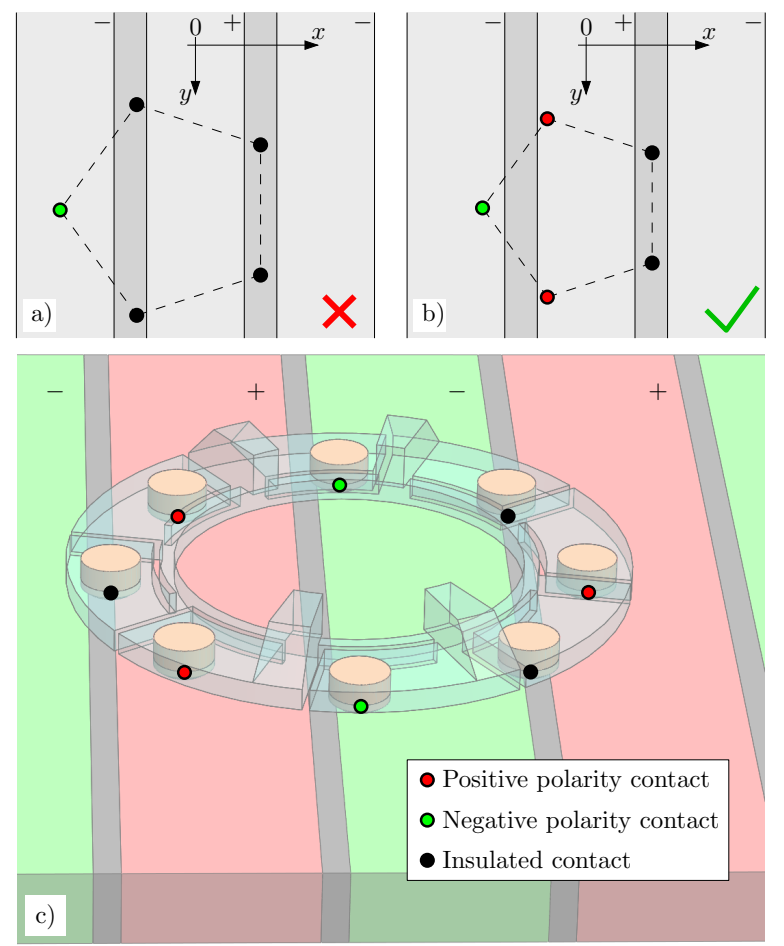

Fig. 2: Contact geometry. In a) a 5-brush geometry is shown. In b) the same is shown at a slightly different scale. In c) our prototype 8-brush geometry is shown. the means for a quantitative approach to the problem, we propose a general framework for the computation of the performance. More in detail, two methodologies are proposed: one discrete, based on set-theory, which assumes there is a clear boundary between conductor and insulation bands; one continuous, which assumes a continuous and differentiable transition between conduction and insulation states. The former methodology is computationally faster, while the latter, although much slower, provides more insight in the robustness of contact close to the boundaries of the conduction bands.

\section{A. Discrete model}

The scheme of Fig. 3 is useful to derive the mathematical framework of the contact problem. The rectangles represent the planar conducting bands whose width is $w$ (light grey color in figure): positive voltage bands are interdigitated with negative ones. They are separated by non-conducting narrow stripes whose width is $v$ (dark grey color in figure). $\Omega$ is the geometric center of the robot, $\langle x, y\rangle$ is the inertial reference frame, $\left\langle x^{\prime}, y^{\prime}\right\rangle$ is the frame linked to the robot, while $\vartheta$ is the angular orientation of the robot. The robot is provided with $n$ sliding contacts $Q_{1}, \ldots, Q_{n}$, named "pins" in the following, that power the battery charging circuit. $\phi_{i}$ and $r_{i}$ are the polar coordinates of $i$-th pin with respect to the robot reference frame. The sliding contacts are placed on a circumference, thus $r_{i}$ is constant. Thanks to the continuity of the conducting bands along the $y$ direction, what it is relevant to the problem is the $x$-coordinate $x_{i}$ of each pin, namely:

$$
x_{i}\left(x_{\Omega}, \vartheta, r_{i}, \phi_{i}\right)=x_{\Omega}+r_{i} \sin \left(\vartheta+\phi_{i}\right)
$$

Let's assume that the origin of the inertial frame is located in the middle of the negative voltage band. Therefore an interval of coordinates $x$ that bounds the negative voltage region is given by the set:

$$
\mathbb{Z}_{l}^{-}=\left\{x \in \mathbb{R}: 2 l(v+w)-\frac{w}{2}<x<2 l(v+w)+\frac{w}{2}\right\}
$$

where $l$ is an index that sequentially numerates the stripes. Without loss of generality, we assume that $l=0$ refers to the negative voltage stripe including the inertial reference frame a)

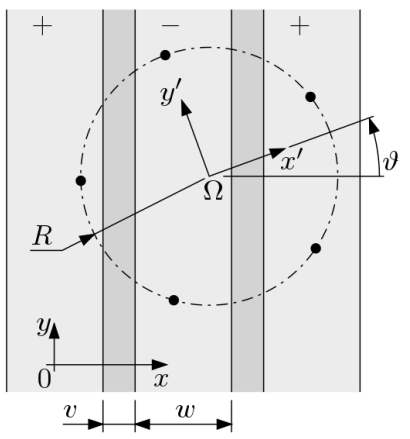

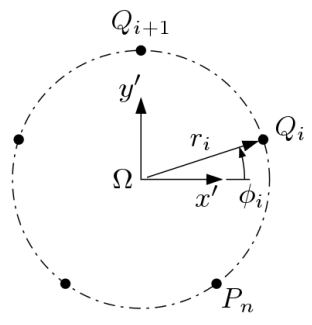

b)
Fig. 3: Definition of the geometry of the system; in a) the positive and negative bands are shown in light grey, whereas dark-grey indicates the insulating material; in b) the $i$ contact points are shown, indicated by the letter $Q$ and the index $i$. 
origin. Likewise, an interval of coordinates $x$ that bounds the positive voltage region is given by the set:

$$
\begin{aligned}
\mathbb{Z}_{l}^{+}= & \{x \in \mathbb{R}: \\
& \left.2 l(v+w)+\frac{w}{2}+v<x<2 l(v+w)+\frac{3}{4} w+v\right\}
\end{aligned}
$$

Note that the integer $l$ can also take negative values. Combining all the sets, it is possible define the non-connected set of values $x$ that defines the negative voltage area and the positive voltage one, respectively

$$
\mathbb{Z}^{-}=\bigcup_{l} \mathbb{Z}_{l}^{-} \quad \text { and } \quad \mathbb{Z}^{+}=\bigcup_{l} \mathbb{Z}_{l}^{+}
$$

In order to define when a pin is in contact with a positive or a negative conductor, let's define the functions:

$$
\begin{aligned}
& f_{i}^{-}=f^{-}\left(x_{\Omega}, \vartheta, r_{i}, \phi_{i}\right)= \begin{cases}1 & \text { if } x_{i} \in \mathbb{Z}^{-} \\
0 & \text { otherwise }\end{cases} \\
& f_{i}^{+}=f^{+}\left(x_{\Omega}, \vartheta, r_{i}, \phi_{i}\right)= \begin{cases}1 & \text { if } x_{i} \in \mathbb{Z}^{+} \\
0 & \text { otherwise }\end{cases}
\end{aligned}
$$

Therefore, when the $i$-th pin is powered by a negative voltage, the Boolean value of $f_{i}^{-}$is 1 (true), otherwise 0 (false). Likewise, when the $i$-th pin is powered by a positive voltage, the Boolean value of $f_{i}^{+}$is true. Note that $f_{i}^{-} f_{i}^{+}=0$ for all $i$. Given the robot geometric parameters $r_{i}, \phi_{i}$, and for a given robot position $\left\{x_{\Omega}, \vartheta\right\}$, the power supply module is powered when there exists a pin $i$ for which $f_{i}^{-}=1$ and, at the same time, there exists another $\operatorname{pin} j$ (with $j \neq i$ ) for which $f_{j}^{+}=1$. Mathematically, this is expressed by the function:

$$
\widetilde{F}\left(x_{\Omega}, \vartheta, \boldsymbol{r}, \boldsymbol{\phi}\right)= \begin{cases}1 & \text { if } \exists i: f_{i}^{+}=1 \wedge \exists j: f_{j}^{-}=1 \\ 0 & \text { otherwise }\end{cases}
$$

where $\boldsymbol{r}=\left\{r_{1}, \ldots, r_{n}\right\}$ and $\phi=\left\{\phi_{1}, \ldots, \phi_{n}\right\}$. A value $\widetilde{F}=$ 1 guarantees power supply for a given pose of the robot on the floor. In order for the robot to reach every point of the working space without losing power supply, it is necessary that $\widetilde{F}=1$ on the entire $\left(x_{\Omega}, \vartheta\right)$ space. Therefore, we can define the function,

$$
F(\boldsymbol{r}, \boldsymbol{\phi})=\min _{x_{\Omega}, \vartheta} \widetilde{F}\left(x_{\Omega}, \vartheta, \boldsymbol{r}, \boldsymbol{\phi}\right)
$$

At this point, we can say that the system is functional if the following is verified:

$$
F(\boldsymbol{r}, \boldsymbol{\phi})=1
$$

However, the function $F$ only provides information about whether the contact occurs or not, but it does not provide any information about the quality of the contact. As an alternative, a different, more descriptive index function can be introduced. Indeed, since each pin-band contact is characterized by an intrinsic resistance that limits the maximum current, it is desirable to describe the performance in terms of the minimum number of connected contacts.

Moreover, by considering that, whatever the geometry $(\boldsymbol{r}, \boldsymbol{\phi})$ and the floor parameters $w$ and $v$, there will necessarily be at least one configuration $\left(x_{\Omega}, \vartheta\right)$ for which at least two pins will be in contact with an insulation band (e.g., see Fig. 2b), the maximum possible number of pins in contact (with either conductive band) will be $n-2$.

The function, called Contact Quality index (CQI), can be defined as follows:

$$
\bar{F}(\boldsymbol{r}, \boldsymbol{\phi})=\min _{x_{\Omega}, \vartheta} \frac{2 \sum_{i} f_{i}^{+}}{n-2}=\min _{x_{\Omega}, \vartheta} \frac{2 \sum_{i} f_{i}^{-}}{n-2}
$$

The rightmost equivalence in Eq. 10 can be demonstrated by noting that there is an inherent symmetry due to the fact that each contact can accept positive or negative polarity indifferently; indeed, every configuration determined by $x_{\Omega}$ has an analogue at $x_{\Omega}+w+v$, but with the polarity flipped for each pin. For example, if the worst configuration for a 8 -contact array has pin 1 on a positive band, pins 2 to 6 on negative bands and pins 7,8 on the insulator, a configuration necessarily exists which has pin 1 on a negative band, and 2 to 6 on positive bands, and the same 7,8 pins on the insulator. We can see that the CQI will be always $\leq 1$; hence:

$$
\bar{F}(\boldsymbol{r}, \phi) \in[0,1]
$$

Finally, the best possible contact configuration occurs when, in the worst robot position and orientation $\left(x_{\Omega}, \vartheta\right)$ case, assuming the pins are even-numbered, the number of pins in contact with positive voltage bands is equal to the number of pins in contact with the negative voltage bands and the pins on insulator bands are exactly two. In case the pins are oddnumbered, the best configuration would be that which shows 2 pins on the insulator bands, while the rest are distributed on the positive and negative bands, with a difference of 1 . Within the same 8-pin example: there always exist at least one position and orientation $\left(x_{\Omega}, \vartheta\right)$ where 2 pins are in contact with an insulator band (there is always a line that passes through two points in a plane); the remaining 6 points are distributed on the polarized bands, three by three. This "best solution" circumstance is expressed when the CQI index approaches 1:

$$
\bar{F}(\boldsymbol{r}, \phi)=1
$$

\section{B. Continuous model}

In order to more accurately characterize the power delivery with respect to the interaction between the robot and the powered floor, we present yet another complete model that includes a fuzzy representation of the conducting bands. This approach has two advantages: it allows to characterize possible irregularities in the bands boundaries, thus providing an approach to the modeling of the possible errors, and it does not involve binary operators or Boolean logic, which can be hard to cope with when using certain numerical optimization methods.

Taking over from the geometrical representation that was described in Fig. 3 and in Section III-A, we can describe the interaction of the individual pin $Q_{i}$ with a specific band as a $\mathbb{C}^{\infty}$ function $\left.p^{+}: \mathbb{R} \mapsto\right] 0,1[$, as shown in Fig. 4a.

To this intent, we propose the function $p^{+}(s)=$ $\varsigma\left(\Lambda, \xi_{a}(s)\right) \varsigma\left(\Lambda, \xi_{b}(s)\right)$, where $\xi_{a}(s)=-s-w / 2$ and $\xi_{b}(s)=$ $s-w / 2$. Note that, in order to define the function $p^{+}$, we introduced the variable $s \in \mathbb{R}$. The function $\varsigma \in \mathbb{C}^{\infty}$ is 
a sigmoid, in this case represented by the logistic curve, as follows:

$$
\varsigma(\Lambda, \xi)=\frac{1}{1+e^{\Lambda \xi}} .
$$

The resulting formulation is as follows:

$$
p^{+}(s)=\frac{1}{\left(1+e^{\Lambda \xi_{a}(s)}\right)\left(1+e^{\Lambda \xi_{b}(s)}\right)}
$$

The function $p^{+}(s)$ is designed to be symmetrical with respect to $s=0$ and presents the following characteristics:

$$
\begin{aligned}
p^{+}(0) & \longrightarrow 1 \\
p^{+}(w / 2) & \longrightarrow \frac{1}{2} \\
p^{+}(w) & \longrightarrow 0
\end{aligned}
$$

An instance of this function is visible in Fig. 4a. The parameter $\Lambda$ influences the rate of change, or "hardness" of the polarity functions.

If we call $\delta s$ the distance from the nominal edge of the band and $\delta p^{+}$the deviation from zero of the polarity at the distance $\delta s$, then we can express Eq. 13 as:

$$
\Lambda=\frac{1}{\delta s} \ln \left(\frac{1}{\delta p^{+}}-1\right)
$$

This allows to define the desired indeterminacy $\varepsilon(\delta s)=\delta p^{+}$ of the bands boundary, and thus assign an adequate value $\Lambda$ to represent it. The same considerations apply to $p^{-}(s)$ as well. For example, in order to have $99 \%$ of $p^{+}$at $1 \mathrm{~mm}$ from the nominal boundary, we would need to assign the value $\Lambda \simeq$ 4595.

Having defined a monopole band, we can now define a corresponding array. This is done by operating on the variable $s$. Indeed, we can consider the array of bands $p_{a}^{+}$and $p_{a}^{-}$as two $\mathbb{C}^{\infty}$ functions themselves, by arguing that:

$$
\begin{aligned}
& p_{a}^{+}(x)=\sum_{k=-N}^{N} p^{+}(x+2 k(w+v)) \\
& p_{a}^{-}(x)=\sum_{k=-N}^{N} p^{-}(x+(2 k+1)(w+v))
\end{aligned}
$$

A formally correct representation would call for $N=\infty$; however, in order to compute the problem numerically, and considering the property of Eq. 17, $N$ can be set as a small finite number, depending on the values of $\Lambda$. At this point, the arrays of positive and negative pole bands are defined formally. By looking at Fig. 3 and Fig. 4b, one can see that the points $Q_{i}$ travel around the surface, each being defined as $Q_{i}=$ $\left\langle x_{Q_{i}}, y_{Q_{i}}\right\rangle^{T}$. We can thus calculate the polarity value of each point, by applying Eq. 19, which yields $p_{a}^{+}\left(x_{Q_{i}}\right)$ and $p_{a}^{-}\left(x_{Q_{i}}\right)$ for all $n$ points $Q_{i}$, thus, from Eq. 1 we can define the Side Polarity functions:

$$
\begin{aligned}
& P^{+}\left(\mathbf{r}, \phi, x_{\Omega}, \vartheta\right)=\sum_{i=1}^{n} p_{a}^{+}\left(x_{Q_{i}}\right) \\
& P^{-}\left(\mathbf{r}, \phi, x_{\Omega}, \vartheta\right)=\sum_{i=1}^{n} p_{a}^{-}\left(x_{Q_{i}}\right)
\end{aligned}
$$

The positive Side Polarity function $P^{+}$can be seen as a measure of the quantity of points that are in contact with the positive bands; by contrast, $P^{-}$shows the quantity related to the negative bands. In fact, in mathematical terms, each point $Q_{i}$ is connected to each existing band, however far. On the other hand, since each band is defined as a rather steep sigmoid, the share to the value $P^{+}$tends to be negligible for distant bands.

Based on these considerations, if we define $\left.\left.P_{\text {th }} \in\right] 0,1\right]$ as a threshold for the $P^{+}$and $P^{-}$functions, then it can be easily seen that the working condition can be written as follows:

$$
P^{+}\left(\mathbf{r}, \phi, x_{\Omega}, \vartheta\right) \geq P_{\mathrm{th}} \wedge P^{-}\left(\mathbf{r}, \phi, x_{\Omega}, \vartheta\right) \geq P_{\mathrm{th}}
$$

or, equivalently:

$$
P\left(\boldsymbol{r}, \boldsymbol{\phi}, x_{\Omega}, \vartheta\right)=\min \left(P^{+}, P^{-}\right) \geq P_{\mathrm{th}}
$$

where $P$ denotes the Total Polarity value of the contact pins on the robot. For example, $P_{\text {th }}=0.5$ leads to similar results to those of the Boolean model described in Section III-A.

Since it stands that $P^{+}$and $P^{-} \in \mathbb{C}^{\infty}$, and thus $P \in \mathbb{C}^{\infty}$, henceforth, since $P^{+}$and $\left.\left.P^{-} \in\right] 0, n\right]$, it can be stated that $P$ provides a smooth measure of the robustness of contact between the powered floor and the contact pins on the robot. Unfortunately, the Total Polarity does not include information on the quality of contact. Therefore, we can define the function $\Pi$ as a measure of the number of positive and negative contacts as follows:

$$
\Pi\left(\boldsymbol{r}, \boldsymbol{\phi}, x_{\Omega}, \vartheta\right)=\frac{2 P\left(\boldsymbol{r}, \boldsymbol{\phi}, x_{\Omega}, \vartheta\right)}{n-2}
$$

which shows perfect balance when $\Pi \approx 1$.

Once we have defined the functions $P\left(\mathbf{r}, \phi, x_{\Omega}, \vartheta\right)$ and $\Pi\left(\mathbf{r}, \phi, x_{\Omega}, \vartheta\right)$, which are in terms of geometry $(\boldsymbol{r}$ and $\phi)$ and configuration $\left(x_{\Omega}\right.$ and $\vartheta$ ), we can proceed to the computation of the associated indices, as done in Eq. 9 and 10, thus having:

$$
\begin{aligned}
F^{*}(\boldsymbol{r}, \boldsymbol{\phi}) & =\min _{x_{\Omega}, \vartheta} P\left(\boldsymbol{r}, \boldsymbol{\phi}, x_{\Omega}, \vartheta\right) \\
\bar{F}^{*}(\boldsymbol{r}, \boldsymbol{\phi}) & =\min _{x_{\Omega}, \vartheta} \Pi\left(\boldsymbol{r}, \boldsymbol{\phi}, x_{\Omega}, \vartheta\right)
\end{aligned}
$$

A critical comparison can be seen in Fig. 5, where plots of $F, \bar{F}, F^{*}$ and $\bar{F}^{*}$ are shown for a specific geometry (regular octahedron) of varying radius, where the two pairs of plots give remarkably similar results. It can be seen that the the continuous method gives more information with respect to the discrete. Furthermore, the binary nature of the discrete method is shown, which is to be expected from the definition of $\tilde{F}$, in 7. Moreover, it is apparent that the continuous method conveys intermediate information related to the interaction of the contact points with the bands boundaries.

\section{Methodology for Regular Polygons}

The mathematical framework described in III can be used to implement a methodology aimed at the design of powered floors where the pins are constrained on the vertices of a regular $n$-gon. In the following paragraphs the main aspects of the methodology will be illustrated, and a practical case study will be described that takes advantage of the methodology. 


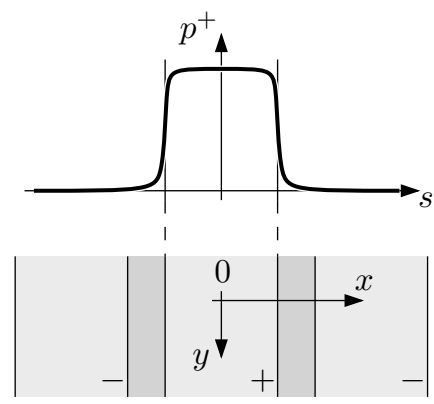

a)

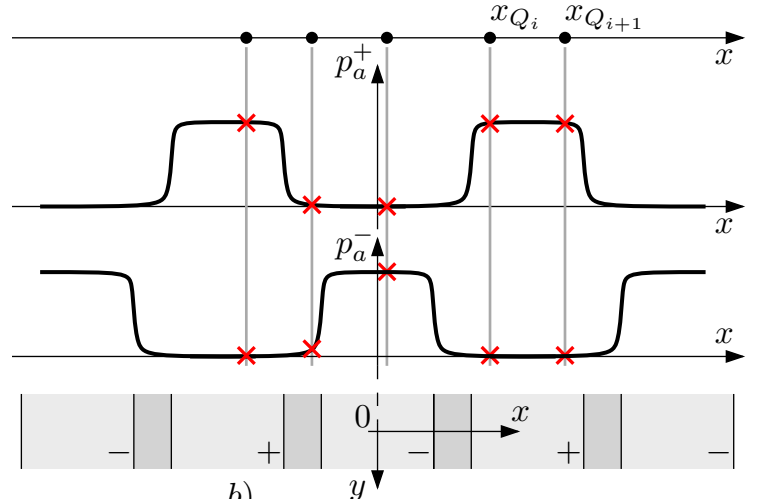

b)

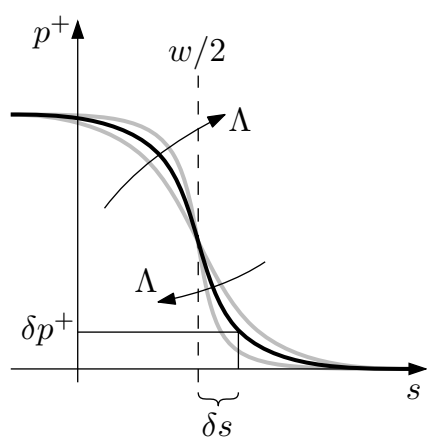

c)

Fig. 4: Definition of the polarity functions. In a), the general positive polarity function $p^{+}$is shown against the variable $s$, highlighting the smooth transition between conducting and insulating bands; in b) the polarity array functions $p_{a}^{+}$and $p_{a}^{-}$are shown for the conductor bands of the system, along with the projections on the $x$-axis of two pins $Q_{i}$ and $Q_{i+1}$; in c) a representation of the fuzzy band-boundary model is shown for a positive polarity $p^{+}$, along with the other main quantities at play.

\section{A. Methodology}

The models described in III, both the discrete and continuous, are general in the sense that they allow for any possible geometry of the contact pins or brushes with respect to the conductor and insulator bands. This geometry is described by the $(\boldsymbol{r}, \phi)$ polar coordinates of points $Q_{i}$. However, by constraining the points $Q_{i}$ to a circumference of radius $R$ as visible in Fig. 3a, a methodology can be described to design the powered floor.

For the $n$-gon of radius $R$, the expressions for the working
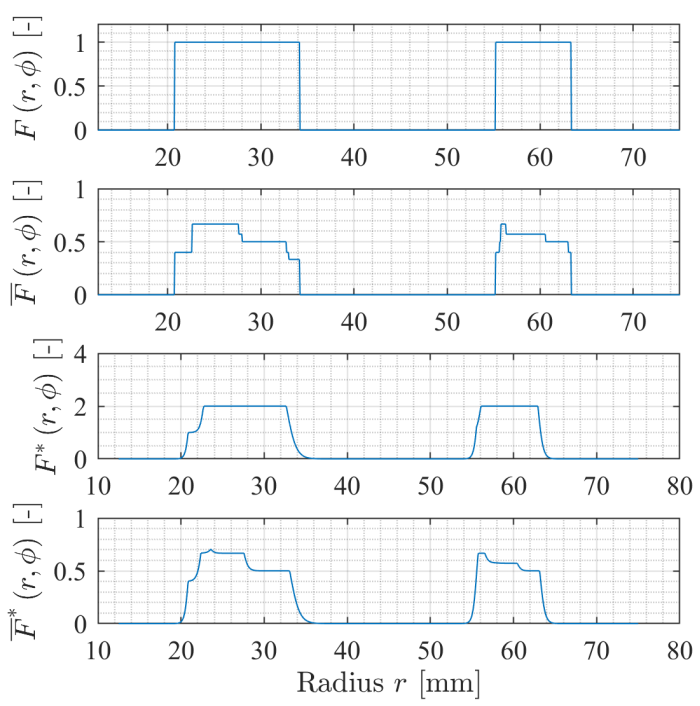

Fig. 5: Performance analysis as a function of the contact pinarray (a regular octahedron) dimension. Comparison between the performance, in terms of polarity functions and polarity balance, with the discrete and continuous methodologies, shown respectively on the top two plots and the bottom two. The parameters used for this analysis are described in detail in Sec. IV-B. condition and CQI can be written as follows:

$$
\begin{aligned}
& F(\boldsymbol{r}, \boldsymbol{\phi})=f(R, n, w, v) \\
& \bar{F}(\boldsymbol{r}, \boldsymbol{\phi})=\bar{f}(R, n, w, v)
\end{aligned}
$$

the same being true for $F^{*}$ and $\bar{F}^{*}$. Each entity is thus 4 dimensional; in principle the problem of finding a solution is that of a maximization of the functions over their domain. However, since this is not within the scope of this work, we will limit the description of a methodology which relies on the graphical analysis of plots.

First, however, we can reduce the domain of the function thanks to the use non-dimensional variables, for example relative to the conductor band width $w$. Thus, we can define the variables $R_{w}=\frac{R}{w}$ and $v_{w}=\frac{v}{w}$, which gives:

$$
\begin{aligned}
& F(\boldsymbol{r}, \boldsymbol{\phi})=f\left(R_{w}, n, v_{w}\right) \\
& \bar{F}(\boldsymbol{r}, \boldsymbol{\phi})=\bar{f}\left(R_{w}, n, v_{w}\right)
\end{aligned}
$$

At this point the design process requires to start by defining one parameter among $R, w$, and $v$, e.g., $w=\tilde{w}$. Then, finite intervals should be determined for the remaining variables, in the example $R, v$, and $n$, as follows:

$$
\begin{aligned}
R_{w} & \in\left[\frac{R_{\text {inf }}}{\tilde{w}}, \frac{R_{\text {sup }}}{\tilde{w}}\right] \subset \mathbb{R}^{+} \\
v_{w} & \in\left[\frac{v_{\text {inf }}}{\tilde{w}}, \frac{v_{\text {sup }}}{\tilde{w}}\right] \subset \mathbb{R}^{+} \\
n & \in\left[n_{\text {inf }}, n_{\text {sup }}\right] \subset \mathbb{N}
\end{aligned}
$$

The functions $F, \widetilde{F}, F^{*}$ and $\widetilde{F}^{*}$ can be computed for each discrete point $\left\langle R_{w}, v_{w}, n\right\rangle$, thus producing a response surface $R S_{R_{w}, v_{w}, n}$ for the working condition function and for the CQI in the discrete and continuous case. Since the variable $n$ is discrete, it is more informative to illustrate $R S_{R_{w}, v_{w}, n}$ as separate plots $R S_{R_{w}, v_{w}}(n)$ with $n \in\left[n_{\text {inf }}, n_{\text {sup }}\right]$. In Fig. 6, the plots can be seen as computed using the discrete method defined in III-A; the continuous method was not used due to the much slower computation; indeed, the computation of the $12100 \times 100$ plots took approximately $36 \mathrm{~h}$ hours on a 
$8 \times 2.80 \mathrm{GHz}, 16 \mathrm{~GB}$ RAM computer, while the continuous methods would have required about seven days on the same machine.

\section{B. Case study}

To show how to use this mathematical framework in practice, let's consider a real case study; the prototype will be based on these values. A mobile robot should move on a surface provided with interdigitated electrodes whose geometrical parameters are $w=25 \mathrm{~mm}$ and $v=3.5 \mathrm{~mm}$. The two main design issues are (1) how many vertices $n$ should the polygon have and (2) what should be its radius $R$ ?

Ideally, the pins should be located at the vertices of a regular octagon due to the structure of the robot, which includes 8 distance sensors around its perimeter. In general, the results illustrated in Fig. 6 suggest that good feasibility envelopes start with $n=7$, which is adequate to meet the geometry of the robot. We can thus refer to the plots of Fig. 5, which are relative to a regular octagon; in particular the top plot shows two complete feasibility windows, the first of which between $R=20.8 \mathrm{~mm}$ and $R=34.2 \mathrm{~mm}$. The second plot confirms the values $(R=20.6 \mathrm{~mm}$ and $R=33.8 \mathrm{~mm}$ ) and provides assurance regarding robustness of contact with values around $F^{*}=2$ in the interval $R=[22.8,32.7] \mathrm{mm}$. It is interesting to note that the polarity balance for this last interval is between $\bar{F}^{*}=0.5$ and 0.7 , which indicates good (albeit not perfect) polarity balance. A hardness value of $\Lambda=4595$ was used,
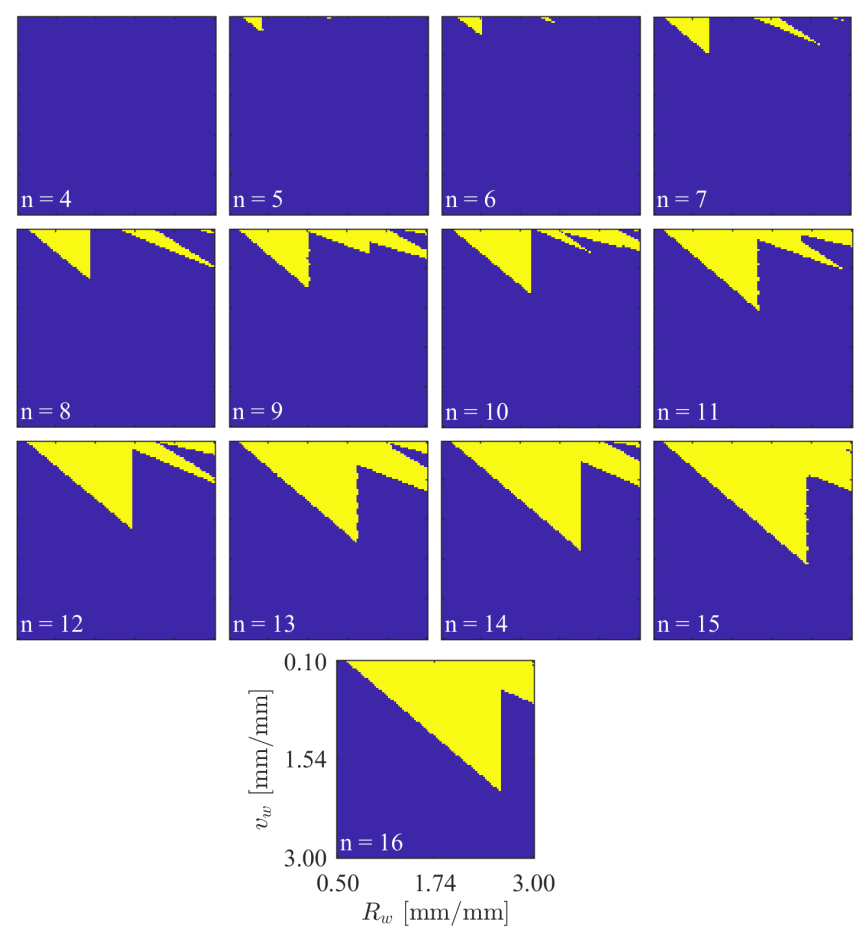

Fig. 6: Response surfaces $R S_{R_{w}, v_{w}}(n)$ of the values of function $F$ for values of $n \in[4,16], R_{w} \in[0.50,3.00]$ and $v_{w} \in[0.10,3.00]$. Yellow indicates feasible configurations. The values of the axes are the same for all plots, and are shown only in case $n=16$ for clarity; the discretization applied to each variable was $\frac{1}{100}$ of the relevant interval.
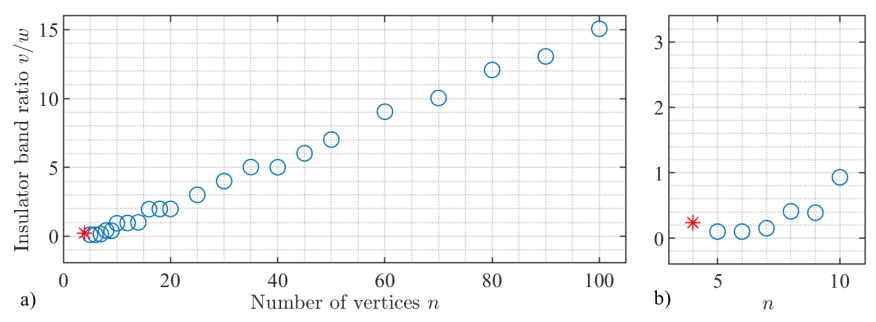

Fig. 7: Relation between the maximum admissible insulation band width $v$ (shown as relative to the conductor bands $w$ ) and the number of vertices of the regular polygons used for the contact pins patterns. The result of Shannon's pattern is shown as a red asterisk, for reference. In a) a complete plot is shown for values from $n=4$ to 100 , whereas in b) only the portion between $n=4$ and 25 is shown, for a better visual comparison to Shannon's pattern.

which assumes an expected deviation of the function $F^{*}$ of $1 \%$ at $1 \mathrm{~mm}$ from the nominal border of the conductor bands. The value of $N$ is set to 10 , which covers the workspace needed for this analysis.

\section{Analysis on the feasibility envelopes}

One aspect which is worth considering is that of the relation between the maximum admissible width $v$ of the insulation band compared to that of the conductor bands $w$. This is especially important due to the fact that a conducting surface such as that used in existing powered floors tends to be covered almost entirely by conducting elements, which proves costly and hard to manufacture and maintain. These generally have an insulator-conductor ratio $\frac{v}{w}$ which tends to be very low.

Referring to Fig. 7, one can appreciate the relation between the ratio $\frac{v}{w}$ and the number of vertices of the polygon in the contact pins pattern which was described in this work. Each data-point represents, for a specific number of vertices $n$, the maximum $\frac{v}{w}$ ratio which produces a feasible configuration, i.e., one where the system is guaranteed to be functional.

This clearly shows that Shannon's pattern-here used as reference-is adequate for a maximum insulation band ratio of $\frac{v}{w}=0.24$; on the other hand, by employing regular polygons of 5 or more vertices produces much higher ratios, resulting in larger insulation bands. Furthermore, this shows that values of $\frac{v}{w} \approx 1$ are feasible with as little as 10 contacts.

Finally, it should be noted that large $\frac{v}{w}$ are especially desirable since, as a rough general estimate, the probability of a short-circuit between two subsequent bands decreases proportionally to the distance $v$ between the bands. Also, large ratios allow for comparatively less conductive material to be positioned on the ground, relative to the size of the contact array, which translates in a less expensive system.

\section{Characterization of non fully functional configuration}

The analysis described in Sec. IV-A determines whether a set configuration guarantees contact at all times, i.e. is "fully functional". Here, we focus on configurations which are not fully functional, by providing a measure of the probability 
that contact does indeed occur. In order to perform this kind of analysis, one can employ a Monte Carlo method. A sample $h$ can be defined as a pair of random values of $x_{\Omega}$ and $\vartheta$ :

$$
t_{h}=\left\langle x_{\Omega}, \vartheta\right\rangle
$$

where the random generated values are bounded according to the geometry of the system, specifically: $x_{\Omega}=[0, w+v[$ and $\vartheta=[0,2 \pi[$.

At this point, one can apply Eq. 7 and find $\tilde{F}_{h}$. The ratio between these and the total number of samples $N$ can be defined as follows:

$$
\eta=\sum_{h} \frac{\tilde{F}_{h}}{N} .
$$

For a sufficiently high number $N$ of samples, the ratio $\eta$ approaches a value which corresponds to the probability $\lambda$ of a configuration $t_{h}=\left\langle x_{\Omega}, \vartheta\right\rangle$ in which there is contact with both positive and negative bands. It goes without saying that the higher $\lambda$ is, the better.

In the following Section, an experimental and numerical approach will be carried out to implement this type of measurement.

\section{PROTOTYPE AND EXPERIMENTAL VALIDATION}

As an example of application of the methodology presented in Section III, we present a prototype of powered floor and brushes which we applied to a commercial small robot (Elisa$3^{1}$ ) tailored to swarm and evolutionary robotics experimentation.

It has to be noted that, being this paper focused on the geometrical aspects of powered floor system design, rather than on the technical ones, we do not consider many factors, such as e.g. efficiency [31], [32], [33], dynamic response [34], [35], wear [36], [37], [38], which of course are very important in real implementations of the design. These factors, moreover, strongly depend on the application, as we may expect that contact technology used e.g. for passengers carrying vehicles will be different from that used for small robots such as the one we discuss in the following.

In Fig. 8, a 3D diagram is shown of the system concept along with some pictures of the actual prototype. As contact pins we used small M1.5 screws with rounded heads, mounted on a polymeric 3D printed flexible support; this is attached via a floating joint to the robot, in order to give both the robot and the contact pins system acceptable adherence to the surface of the powered floor. The powered floor itself consists in a planar sheet of plastic on top of which strips of conducting copper tape are arranged according to the specifications which are given in Section IV-B. Considering these parameters, and using Eq. 26, the value for the radius of the contact array was selected at $r=32 \mathrm{~mm}$, while the number of contact points was determined to be $n=8$, thus defining a regular octagon. The plots in Fig. 5 show that these values are within the feasibility envelope. The other considerations highlighted in Sec. IV-B

${ }^{1}$ http://www.gctronic.com/doc/index.php/Elisa-3 apply. In fact, we found that alternating the contact point and the sensor produced the best results, leaving the area in front of the IR sensor free.

\section{A. Power supply circuit}

In order to implement our power supply system in the robot, we added a set of diodes in a configuration which reminds of the 3-phase or 6-phase bridge systems commonly used in $50 \mathrm{~Hz}$ and $60 \mathrm{~Hz}$ power rectifier units; the output of the rectifier is simply connected to the USB power input of the robot. In Fig. 9a the circuit diagram in the case of 8 sliding contacts is shown.

\section{B. Contact resistance measurements}

The quality of contact between the brushes and the conducting bands is a critical issue; it impacts the transfer of power to the robot, both in terms of power losses and in terms of intermittent delivery. Wear of the bands and/or of the brushes can negatively affect both of these aspects. Although there are several options to keep these problems at bay, all mitigation efforts tend to affect power management and could degrade the overall performance.

A set of measurements has been performed in order to evaluate the resistance of pairs of contacts in series, i.e., considering the case of one contact on a positive band and a contact on the negative one; we shall refer to this resistance in the following as "total resistance". This is the most unfavourable situation; indeed, when two or more contacts are working on the same band or on bands with the same polarity, current is of course distributed and the overall resistance decreases.

Measures have been acquired according to the diagram reported in Fig. 9b. We bypassed the circuit between two contacts, set a current $I$ and measured the voltage drop $V$ across the two bands touched by the contacts, so that we get $R=V / I$. The advantage of this solution with respect to measuring the resistance of a single contact using a multimeter is that bench instrumentation can be used, even with a moving robot. A $120 \mathrm{~mA}$ current source (this is the order of magnitude of the average current absorbed by the robot) has been realized using a voltage source $(\mathrm{Vdc})$ and a $100 \Omega 2 \mathrm{~W}$ series resistance (R1). It might seem that a current generator could perform better than a voltage generator with a series resistance; however, common bench power supplies normally have far from ideal time domain response while operating as current source, while as voltage generator they can provide very stable voltages. The resistance R1, being non-infinite, introduces an error, which however is small as the value of $\mathrm{R} 1$ is much larger than the contact resistances (a few ohms). Two $1 \mathrm{~N} 4728$ $3.3 \mathrm{~V} 1 \mathrm{~W}$ Zener diodes have been added in order to protect the input of the acquisition system (a digitizing oscilloscope) in the case of missing or very poor contact; otherwise, with no voltage drop on $\mathrm{R} 1$, the full $\mathrm{Vdc}$ voltage would reach the oscilloscope.

When the robot is still, measured total resistance is around $1 \Omega-2 \Omega$. The standard deviation is small, roughly $0.05 \Omega-$ $0.07 \Omega$, but if the robot is re-positioned the measures values may change, as it can be seen in Fig. 10a. 


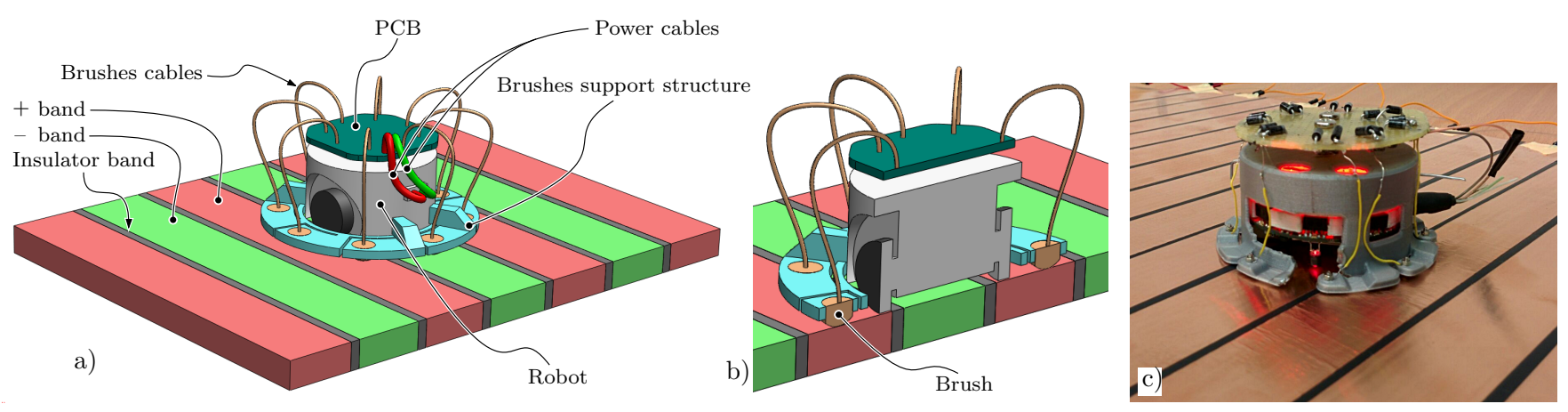

Fig. 8: Conceptual diagram of the powered floor, robot and pictures of the implemented prototype. In a) the main components of the robot and the brushes system are shown; in b) a detailed cut-section of the assembly illustrates the interaction between the polarized bands and the brushes. In c) the experimental implementation is shown.

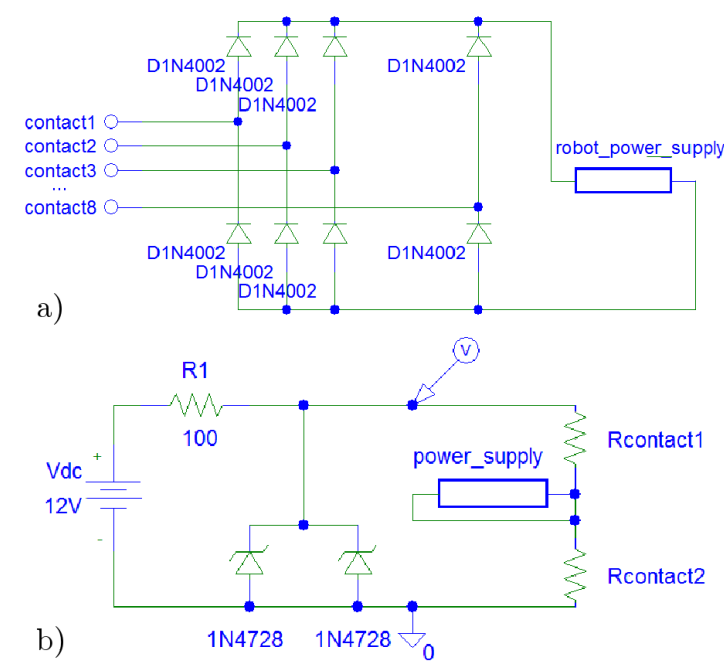

Fig. 9: Electrical diagrams of the prototype and measurement system. In a) the schematic diagram of the rectifier circuit is shown, while in $b$ ) the diagram of the resistance measurement circuit is illustrated.

It is more interesting to examine the total resistance during robot motion. In Fig. 10b an example is shown which has been obtained while moving the robot so that no change in bands occur during the test; sampling frequency is $1.25 \mathrm{MS} \mathrm{s}^{-1}$. It may be noticed that larger values and larger variations are obtained; indeed, measured values are around $3 \Omega-5 \Omega$ with a standard deviation of $0.4 \Omega-0.7 \Omega$. This is not surprising, however, as contacts have been realized using common screws which slide on copper tape: both oxidation and wear can worsen the quality of the contact. It must also be added however that, as the robot is equipped with a regulated power supply and a battery, possible variation in the supply voltage do not have a significant effect on the performances of the system.

\section{Contact statistical analysis results}

It is useful to have a probabilistic measure of the likelihood that loss of contact happens for a set configuration. Following the methodology laid out in Sec. IV-D, two experiments, one
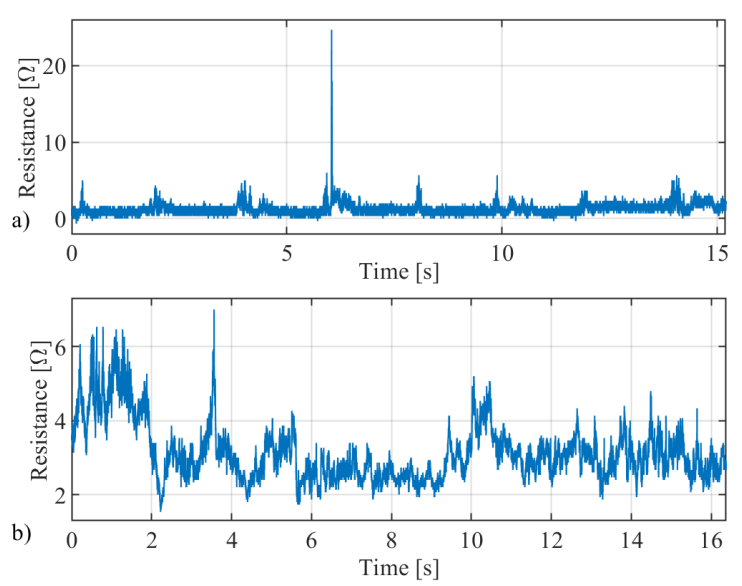

Fig. 10: Experimental results. In a) a measurement of temporal variations of the total contact resistance is shown: the robot is briefly displaced each $2 \mathrm{~s}$. It may be noticed that during displacement the resistance increases significantly; in one case the contact is almost lost and there is the intervention of the diode protection. In b) temporal variations of the total contact resistance during robot motion are shown.

numerical and one physical, were designed and performed. The number $n$ of contacts selected for the polygons in these experimental campaign are $3,4,6$, and 8 .

The numerical experiment is based on a Monte Carlo random approach to generate the pairs $t_{h}$. In Fig. 11 the accuracy in the determination of an estimate of $\eta$ is shown in relation to the number of samples $N$. This analysis was performed by generating 100 populations of an increasing number $N$ of samples of $\eta$ using Eq. 36. As expected, the plots in Fig. 11b in particular show that the standard deviation dramatically decreases for larger values of $N$. Indeed, for a value of $N=10^{4}$ samples, $\sigma_{\eta}=0.0044$, calculated for a 3 -gon configuration.

The physical experiment consisted in the replication on a smaller scale of the Monte Carlo simulation. The robot was commanded to position itself in a set of 100 different positions with a 3-, 4-, 6- and 8-sided regular polygons contact arrays. The control sequence consists in a straight drive of random 


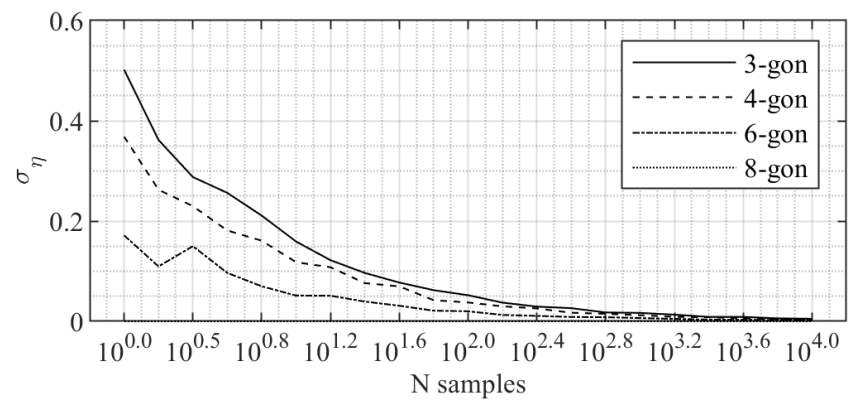

Fig. 11: Monte Carlo simulation. Influence of the number of samples $N$ on the accuracy of the estimation of $\eta$. A plot of the standard deviation of the populations of 100 elements is shown for each number of sample $N$.

TABLE I: Determination of $\eta$ using the numerical $\left(\eta_{\text {num }}\right)$, with $N=10^{4}$, and experimental $\left(\eta_{\text {exp }}\right), N=10^{2}$, methodologies.

\begin{tabular}{cccc}
\hline$n$ & $\eta_{\text {num }}$ & $\eta_{\exp }$ & $\left|\eta_{\text {num }}-\eta_{\exp }\right|$ \\
\hline 3 & 0.5169 & 0.5300 & 0.0131 \\
4 & 0.8186 & 0.8300 & 0.0114 \\
6 & 0.9642 & 0.9800 & 0.0158 \\
8 & 1.0000 & 1.0000 & 0.0000 \\
\hline
\end{tabular}

length between 0 and $50 \mathrm{~mm}$, followed by a stand-still rotation of a random quantity between 0 and $2 \pi$. This process repeats 100 times for each contact array. At the end of each rotation, the system acquires the polarity of each pin and stores it in a log file. These measurements allow the computation of the quantity $\tilde{F}$, as defined in Eq. 7. At this point, it is possible to calculate the value of $\eta$ from experimental data.

The results of the numerical simulation using the Monte Carlo method and the experimental results are shown in Table I; it may be noticed that they show very good similarity. The value of $\eta$ for an 8 -gon is 1.00 , signifying that, as expected, this configuration provides contact at all times, i.e., the probability of a functional pose is $100 \%$. On the other hand, as the number of vertices decreases, so does the probability of a functional pose, which is fully compatible and, in fact, supports the results shown in Fig. 6.

\section{CONCLUSION}

In the field of mobile robotics, the topic of power delivery is especially important, given the rapid propagation of the technology and the intrinsic limitations of batteries.

In this paper, we followed-up on the concept of poweredfloors, consisting in a surface of interdigitated conducting bands and a contact array of specific shape on the mobile system. We presented a complete framework for the analysis and design of these systems, introducing two methodologies (one discrete, one continuous) to simulate the interaction between the floor and the contact array. The continuous method provides a way to account for uncertainties and positioning errors of the contact points relative to the bands. In this work we consider contact arrays of the shape of regular polygons, but the methodology can be extended to arbitrary distributions of contact points. Performance was measured thanks to two quantities: a functionality condition $F$ (discrete) and $F^{*}$ (continuous) and a Contact Quality index (CQI and Continuous CQI, i.e., CCQI).

In the paper, we showed a comprehensive analysis on the functionality envelopes given by $F$ for $n \in[4,16]$. In fact, starting from $n=5$ vertices, it is always possible to find a regular polygon that provides a reliable powerdelivery connection. Furthermore, we illustrated the relation between $n$ and the insulator band/conductive band ratio $v / w$ and we compared our regular polygons with the configuration first introduced by C. Shannon in 1950; we find that, for $n \geq 8$, regular polygons consistently outperform his nonregular $n=4$ solution.

Finally, a prototype of an octagon shaped contact array and powered floor was designed, and was applied to a commercially available Elisa-3 robot. As expected, the associated experimental campaign showed that the power delivery system does indeed provide electrical continuity between the floor and the contact array at all times.

Going forward, more in-depth analysis should be performed on non-polygonal distribution of the contact arrays. Furthermore, the interactions between the brushes and the bands should be investigated beyond the resistance measurements which have been presented in this paper.

\section{REFERENCES}

[1] S. Rezvanizaniani, Z. Liu, Y. Chen, and J. Lee, "Review and recent advances in battery health monitoring and prognostics technologies for electric vehicle (ev) safety and mobility," Journal of Power Sources, vol. 256, pp. 110-124, 2014.

[2] A. Shing and P. Wong, "Wear of pantograph collector strips," Proceedings of the Institution of Mechanical Engineers, Part F: Journal of Rail and Rapid Transit, vol. 222, no. 2, pp. 169-176, 2008.

[3] T. Ding, G. Chen, J. Bu, and W. Zhang, "Effect of temperature and arc discharge on friction and wear behaviours of carbon strip/copper contact wire in pantograph-catenary systems," Wear, vol. 271, no. 9-10, pp. 1629-1636, 2011.

[4] L. Pastena, "A catenary-free electrification for urban transport: An overview of the tramwave system," IEEE Electrification Magazine, vol. 2, no. 3, pp. 16-21, 2014.

[5] Y. Mei, Y.-H. Lu, Y. Hu, and C. Lee, "A case study of mobile robot's energy consumption and conservation techniques," vol. 2005, 2005, pp. 492-497.

[6] A. Sadrpour, J. Jin, and A. Ulsoy, "Mission energy prediction for unmanned ground vehicles using real-time measurements and prior knowledge," Journal of Field Robotics, vol. 30, no. 3, pp. 399-414, 2013.

[7] V. Rajan, A. Nagendran, A. Dehghani-Sanij, and R. Richardson, "Tether monitoring for entanglement detection, disentanglement and localisation of autonomous robots," Robotica, vol. 34, no. 3, pp. 527-548, 2016.

[8] J. Wang, M. Hu, C. Cai, Z. Lin, L. Li, and Z. Fang, "Optimization design of wireless charging system for autonomous robots based on magnetic resonance coupling," AIP Advances, vol. 8, no. 5, 2018.

[9] M. Yang, G. Yang, E. Li, Z. Liang, and H. Lin, "Modeling and analysis of wireless power transmission system for inspection robot," 2013.

[10] S. Li and C. Mi, "Wireless power transfer for electric vehicle applications," IEEE Journal of Emerging and Selected Topics in Power Electronics, vol. 3, no. 1, pp. 4-17, 2015.

[11] J. Shin, S. Shin, Y. Kim, S. Ahn, S. Lee, G. Jung, S.-J. Jeon, and D.-H. Cho, "Design and implementation of shaped magnetic-resonance-based wireless power transfer system for roadway-powered moving electric vehicles," IEEE Transactions on Industrial Electronics, vol. 61, no. 3, pp. 1179-1192, 2014.

[12] F. Musavi, M. Edington, and W. Eberle, "Wireless power transfer: A survey of ev battery charging technologies," 2012, pp. 1804-1810.

[13] J. Huh, S. Lee, W. Lee, G. Cho, and C. Rim, "Narrow-width inductive power transfer system for online electrical vehicles," IEEE Transactions on Power Electronics, vol. 26, no. 12, pp. 3666-3679, 2011. 
[14] A. Marinescu, G. Rosu, L. Mandache, and O. Baltag, "Achievements and perspectives in contactless power transmission," 2018, pp. 638-645.

[15] Y. Jang, "Survey of the operation and system study on wireless charging electric vehicle systems," Transportation Research Part C: Emerging Technologies, vol. 95, pp. 844-866, 2018.

[16] S. Hasanzadeh and S. Vaez-Zadeh, "A review of contactless electrical power transfer: Applications, challenges and future trends [pregled slanja u području bezkontaktnog prijenosa električne energije: Primjene, izazovi i trendovi]," Automatika, vol. 56, no. 3, pp. 367-378, 2015.

[17] M. Rappaport and C. Bettstetter, "Coordinated recharging of mobile robots during exploration," vol. 2017-September, 2017, pp. 6809-6816.

[18] S. Nakamura, S. Hashimoto, and H. Hashimoto, "Preliminary development of an energy logistics as a new wireless power transmission method," in IECON 2013 - 39th Annual Conference of the IEEE Industrial Electronics Society, Nov 2013, pp. 7843-7848.

[19] S. Seriani, P. Gallina, and A. Wedler, Dynamics of a tethered rover on rough terrain, ser. Mechanisms and Machine Science, 2017, vol. 47.

[20] _ "A modular cable robot for inspection and light manipulation on celestial bodies," Acta Astronautica, vol. 123, pp. 145-153, 2016.

[21] D. Poljanec, M. Kalin, and L. Kumar, "Influence of contact parameters on the tribological behaviour of various graphite/graphite sliding electrical contacts," Wear, vol. 406-407, pp. 75-83, 2018.

[22] M. Grandin and U. Wiklund, "Wear and electrical performance of a slip-ring system with silver-graphite in continuous sliding against pvd coated wires," Wear, vol. 348-349, pp. 138-147, 2016.

[23] R. Watson, S. Ficici, and J. Pollack, "Embodied evolution: Distributing an evolutionary algorithm in a population of robots," Robotics and Autonomous Systems, vol. 39, no. 1, pp. 1-18, 2002.

[24] J. Klingner, A. Kanakia, N. Farrow, D. Reishus, and N. Correll, "A stick-slip omnidirectional powertrain for low-cost swarm robotics: Mechanism, calibration, and control," 2014, pp. 846-851.

[25] F. Arvin, S. Watson, A. Turgut, J. Espinosa, T. Krajník, and B. Lennox, "Perpetual robot swarm: Long-term autonomy of mobile robots using on-the-fly inductive charging," Journal of Intelligent and Robotic Systems: Theory and Applications, pp. 1-18, 2017.

[26] S. Martel, M. Sherwood, C. Helm, W. G. de Quevedo, T. Fofonoff, R. Dyer, J. Bevilacqua, J. Kaufman, O. Roushdy, and I. Hunter, "Three-legged wireless miniature robots for mass-scale operations at the sub-atomic scale," in Proceedings 2001 ICRA. IEEE International Conference on Robotics and Automation (Cat. No.01CH37164), vol. 4, 2001, pp. 3423-3428.

[27] S. Seriani, L. Scalera, A. Gasparetto, and P. Gallina, A new family of magnetic adhesion based wall-climbing robots, ser. Mechanisms and Machine Science, 2019, vol. 68.

[28] S. Koos, J.-B. Mouret, and S. Doncieux, "The transferability approach: Crossing the reality gap in evolutionary robotics," IEEE Transactions on Evolutionary Computation, vol. 17, no. 1, pp. 122-145, 2013.

[29] J. C. Bongard, "Evolutionary robotics," Communications of the ACM, vol. 56, no. 8, pp. 74-83, 2013.

[30] E. Medvet, S. Seriani, A. Bartoli, and P. Gallina, "Design of powered floor systems for mobile robots with differential evolution," in International Conference on the Applications of Evolutionary Computation (Part of EvoStar). Springer, 2019, pp. 19-32.

[31] T. Ueno, K. Kadono, and N. Morita, "Influence of surface roughness on contact voltage drop of electrical sliding contacts," in Electrical Contacts - 2007 Proceedings of the 53rd IEEE Holm Conference on Electrical Contacts, Sep. 2007, pp. 200-204.

[32] T. G. Engel, J. M. Neri, and M. J. Veracka, "The velocity and efficiency limiting effects of magnetic diffusion in railgun sliding contacts," in 2008 14th Symposium on Electromagnetic Launch Technology, June 2008, pp. $1-5$.

[33] F. Guo, W. Jia, Z. Chen, Z. Wang, F. Yin, Y. Liu, and Y. Xue, "Experimental research on current-carrying and friction characteristics of sliding electrical contact," in 2010 Proceedings of the 56th IEEE Holm Conference on Electrical Contacts, Oct 2010, pp. 1-6.

[34] Q. Zhang, J. Li, R. Cao, P. Liu, and S. Li, "Simulation and test research of copper-aluminum sliding pair dynamic contact performance," in 2012 16th International Symposium on Electromagnetic Launch Technology, May 2012, pp. 1-4.

[35] S. A. Romanishina, D. Y. Katyuk, V. S. Deeva, and S. M. Slobodyan, "Dynamics layer of the sliding contact collector elements," in 2015 IEEE 35th International Conference on Electronics and Nanotechnology (ELNANO), April 2015, pp. 116-118.

[36] Chen Zhonghua, Hui Lichuan, Wang Tiejun, and Guo Fengyi, "Modeling study of the amount of wear in sliding electric contact," in 26th International Conference on Electrical Contacts (ICEC 2012), May 2012, pp. 146-150.
[37] K. Mashimo, H. Nishikubo, Y. Ishimaru, Y. Okuno, and S. Kawata, "Prediction of wear volume on sliding contacts using cellular automata," in 2015 IEEE 61st Holm Conference on Electrical Contacts (Holm), Oct 2015, pp. 408-413.

[38] Z. Chen, G. Sun, G. Shi, and L. Hui, "Study on friction and wear of sliding electrical contact of pantograph-catenary system under fluctuating compressive load," in 2018 IEEE Holm Conference on Electrical Contacts, Oct 2018, pp. 399-405.

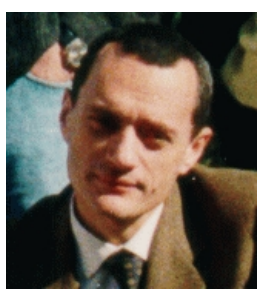

Sergio Carrato graduated in Electronic Engineering at the University of Trieste; he then worked at Ansaldo Componenti and at Sincrotrone Trieste, in the field of electronic instrumentation for applied physics, and received the Ph.D. in Signal Processing from the University of Trieste. Later he joined the Department of Electronics of the same University, where he is currently associate professor of Electronic Devices. His research interests include electronics and signal processing, and, in particular, image and video processing, also for forensic applications, and the development of advanced instrumentation for experimental physics laboratories.

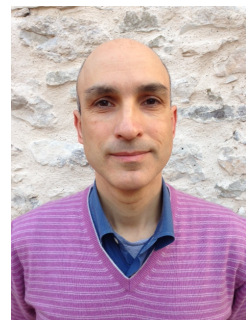

Paolo Gallina Paolo Gallina is currently associate professor of Applied Mechanics at the Department of Engineering and Architecture, University of Trieste, Trieste (Italy). He was visiting professor at the Ohio University in 2000/1. In 2002 he implemented a hands-on Mechatronics Laboratory for students in Engineering. In 2003 he implemented a Robotics Laboratory where he carries out his main research in robotics. He was head of the Council for Students in Mechanical Engineering Degree from 2004 to 2008 $\mathrm{He}$ is head of the Council for Students in Industrial Engineering. He is the Director of the Master in Robotics at the University of Trieste. His interests are in vibrations, human-machine interfaces and robotics.

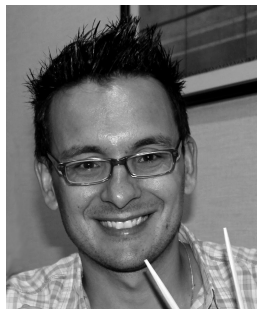

Eric Medvet received the degree in Electronic Engineering cum laude in 2004 and the $\mathrm{PhD}$ degree in Computer Engineering in 2008, both from the University of Trieste, Italy. He is currently an Assistant Professor in Computer Engineering at the Department of Engineering and Architecture of University of Trieste, Italy, where he leads the Evolutionary Robotics and Artificial Life lab (ERALlab) and is the co-head of the Machine Learning Lab. His research interests include Genetic Programming and Machine Learning applications.

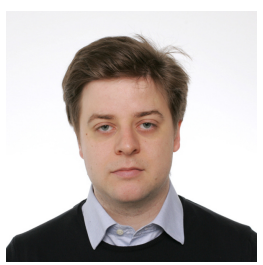

Stefano Seriani born in Trieste (Italy) in 1986, received his B.E. (2010) and his M.Sc in mechanical engineering (2012) from the University of Trieste, Italy. He received his $\mathrm{PhD}$ in April 2016 at the University of Trieste, Italy. In 2016 he was research fellow at the Institute of Robotics and Mechatronics of the German space agency (DLR). He is now research fellow at University of Trieste. His research interests include space robotics, applied mechanics, and computer-vision. 